\title{
Portal Vein Diameter by Ultrasonography as a non-Invasive Predictor of Esophageal Varices
}

\author{
Amal Basnet ${ }^{1}$, Jiwan Thapa ${ }^{1}$, Kalyan Sapkota ${ }^{2}$, Anjana KC ${ }^{1}$ \\ ${ }^{1}$ Department of Medicine, National Academy of Medical Sciences, Bir Hospital, Nepal \\ ${ }^{2}$ Department of Medicine, Bharatpur Hospital, Nepal
}

\section{ABSTRACT}

Esophageal varices are one of the most serious consequence of portal hypertension in patients with liver diseases If undetected or left untreated, it can result in massive upper gastrointestinal bleed and can be fatal. Therefore, a timely detection and management of the varices in cirrhotic patient is a must. Several studies have evaluated possible noninvasive markers of esophageal varices to reduce the number of unnecessary endoscopies in patients with cirrhosis but without varices. Endoscopic surveillance of esophageal varices in patients with liver cirrhosis is expensive for the health system and uncomfortable for the patients. Recently, non-invasive ultrasound-based parameters seem to offer valuable information about the status of esophageal varices and thus challenge the need for repetitive endoscopic monitoring. In this study, our aim was to develop a predictive model using an independent risk factor for the presence of varices in the enrolled patients. This is a cross-sectional study. The study analyzed 50 patients with liver diseases from February 2014 to January 2015. All the patients with cirrhosis of either gender except those with hepatocellular carcinoma and previous surgical or medical intervention for portal hypertension were enrolled in the study. Ultrasound of the abdomen was done in all cases to note the portal vein diameter. The varices visualized were graded endoscopically. Data entry was done in SPSS version 20 and statistical analysis was done with Chi Square test. Total 50 patients were enrolled in the test. Varices was present in 47 $(96 \%)$ of the patients. Small varices were present in $13(26 \%)$ and large varices was present in $34(68 \%)$ of patients. For the patients with small varices, the mean portal vein diameter was $13.23 \mathrm{~mm}$ and for those with large varices, the mean portal vein diameter was $16.83 \mathrm{~mm}$. The above results signify that the presence of varices was associated with the mean portal vein diameter of $13.23 \mathrm{~mm}$ or more and the result was statistically significant with the p value of 0.024 Measurement of portal vein diameter by ultrasonography can be used as a non-invasive predictive indicator of the development of gastroesophageal varices in patients with portal hypertension.

Keywords: Cirrhosis, Esophageal varices, Portal vein diameter, Portal hypertension

\section{INTRODUCTION}

One of the major cause of morbidity and mortality in Nepal is liver cirrhosis though the exact burden of the disease is still not completely measured. Alcohol consumption is socially acceptable in our concept and seems to be the major cause of liver cirrhosis. A retrospective study done by Mishra et al, in 2002 in Bir hospital showed that chronic alcohol consumption related Liver Disease account for $31.4 \%$ of outpatient and $41.4 \%$ of inpatient visits in the hepatology department [1].The prevalence of esophageal varices in liver cirrhosis ranges around $50 \%$ and $80 \%[2]$.

* Correspondence: Dr. Amal Basnet, Department of Internal Medicine, National Academy of Medical Sciences, Bir Hospital. Kathmandu, Nepal. E-mail: amalbasnet001@gmail.com, Telephone: - +977-9841677213
The high prevalence of esophageal varices on endoscopy is probably due to the late presentation of patients after signs and symptoms of portal hypertension were present [3]. Portal hypertension leads to dilatation of portal vein, splenomegaly, and formation of portal systemic collaterals (esophageal varices) at different sites. These gastro-esophageal varices are

responsible for the main complications of portal hypertension and massive upper gastrointestinal bleeding [4]. Gastroduodenoscopy is still considered the best way for the diagnostic evaluation and therapeutic management of the varices. However, the procedure is technically challenging for the rural health systems and procedurally unpleasant for the patient, especially so when it has to be repeated frequently, within the framework of a screening program. Therefore, an alternative noninvasive 
diagnostic parameter is required for the surveillance of the varices in patients with portal hypertension so that gastroduodenoscopy need only be used in those patients who require it for therapeutic purpose [5]. Despite the advantages of endoscopy, it is still an unpleasant and expensive invasive method. It also carries the risk of bleeding due to manipulation. Ultrasound findings in portal system (portal vein size) could predict both the presence of varices and risk of variceal bleeding. It can be used for diagnosis as well as long-term clinical monitoring of patients with portal hypertension [6]. In this study, we investigated a possible predictive value of certain ultrasound parameters of portal vein for the risk of bleeding esophageal varices with liver cirrhosis and portal hypertension by comparing ultra-sonographic and upper gastrointestinal endoscopic findings. If ultra-sonographic findings are reliable, patients can avoid repeated, expensive procedure(endoscopy) for detection of bleeding esophageal varices. Clinicians can measure portal vein size by ultrasonography and take proper steps to manage patients cost effectively. Also in rural areas in Nepal where a lot of cirrhosis cases are prevalent and endoscopic expertise and facilities are not available, ultrasonography can be used to detect cases at high risk of variceal bleeding. As earlier studies, have demonstrated the relationship between portal vein size and the presence of esophageal varices, we want to reconfirm these findings.

\section{METHODOLOGY}

The observational, cross-sectional, hospital based study was carried out at Bir Hospital, Mahabouddha, Kathmandu from February 2014 to January 2015.The total of 50 patients with age equal to or more than $15 y$ rs who is a diagnosed case of liver cirrhosis or who meet the criteria of liver cirrhosis based on clinical, biochemical and ultra sonographic findings were included in the study. However, the patients who had already undergone endoscopic or surgical intervention for portal hypertension or those with evidence of hepatocellular carcinoma on ultrasonography were excluded from the study.

Details of the patient including demographics, relevant history and physical examination findings, were entered in the questionnaires prepared for individual patients. As per the hospital protocol, patient's blood was drawn by the paramedic staff and send for baseline investigations including liver function tests. Additional tests including ECG and Chest $\mathrm{X}$ ray were done depending on the individual patient's health condition. All patients in the sample were also sent for ultrasonological evaluation of the abdomen for evidence of liver Cirrhosis. All the patients were examined with 3.6 and $6.2 \mathrm{MHz}$ curvilinear and linear probes of Toshiba model Aplio 400 ultrasound machine. Examination was carried out after a minimum of 8 hours overnight fasting. With the patient in the supine position, assessment of the size, echo pattern of the liver and the diameter of portal vein was made by gray scale sonography using a combination of subcostal and intercostal approaches. Liver span was measured in the midclavicular line. The normal liver's echo pattern is homogeneous. A diffuse coarse echo pattern with surface irregularity and nodularity was considered abnormal. Collaterals was assessed using color Doppler at the splenic hilum, at the gastroesophageal junction (esophageal varices), in the ligamentum teres (for recanalized umbilical vein) and in the gallbladder bed. Endoscopy was performed on the next morning of admission for bleeding varices. For other cirrhotic patients, routine endoscopy was performed after obtaining date for endoscopy. Patient was kept on nothing per oral status overnight and was sent for endoscopy on given date. Varices are classified as small $(\leq 5 \mathrm{~mm}$ diameter) or large ( $>5 \mathrm{~mm}$ diameter $)$ when assessed with full insufflation [7].

\section{Data Collection and Statistical Analysis}

Data were collected on a structured Performa covering the relevant subjects of the study. Data on clinical status of the study participant were collected via chart extraction of patients admitted to Gastroenterology department and Liver unit with a diagnosis of cirrhosis. Data entry was done in Statistical Packages for the Social Sciences version 20. All categorical data were expressed in percent and absolute number. All numerical continuous data were expressed in mean \pm SD. The data analysis was done using Statistical Packages for the Social Sciences version 20. Appropriate statistical test was carried out for testing the significance. All tests were analyzed with a $95 \%$ confidence interval and a $\mathrm{P}$ value of $<0.05$ was considered significant.

\section{RESULTS}

The results showed that out of 50 patients evaluated, varices were absent in 6\% (3) of patients with cirrhosis. Most the cases 68\% (34) had large esophageal varices and 26\% (13) of cases had small varices (figure 1). Most cases had portal vein diameter in the range 13 to $14.9 \mathrm{~mm}(40 \%), 22 \%(11)$ had portal vein diameter more than $15 \mathrm{~mm}, 26 \%$ (13) cases had portal vein diameter in the range 11 to 12.9 $\mathrm{mm}$ and $12 \%(6)$ had portal vein diameter less than $11 \mathrm{~mm}$ (figure 2). It was found that the mean diameter of the portal vein was $10.4 \mathrm{~mm}$ in the patients without varices. For the patients with small varices, the mean portal vein diameter was 13.23 $\mathrm{mm}$ and for those with large varices, the mean portal vein diameter was $16.83 \mathrm{~mm}$ (Table 1). The above results signify that the presence of varices was associated with the mean portal vein diameter of 
$13.23 \mathrm{~mm}$ or more (Figure 3) and the result was statistically significant with the $\mathrm{p}$ value of 0.024 .

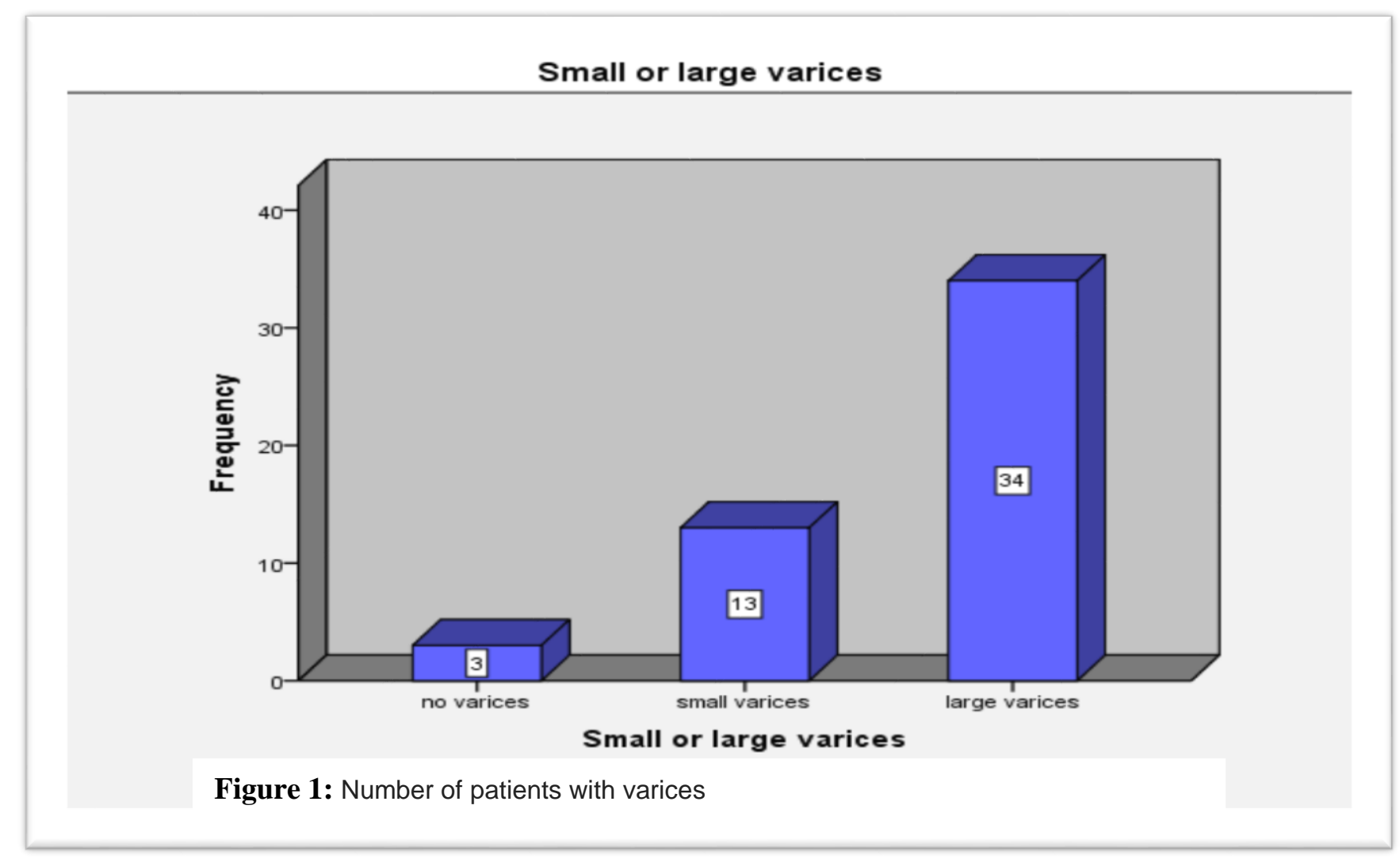

\section{RangeOfPVD}

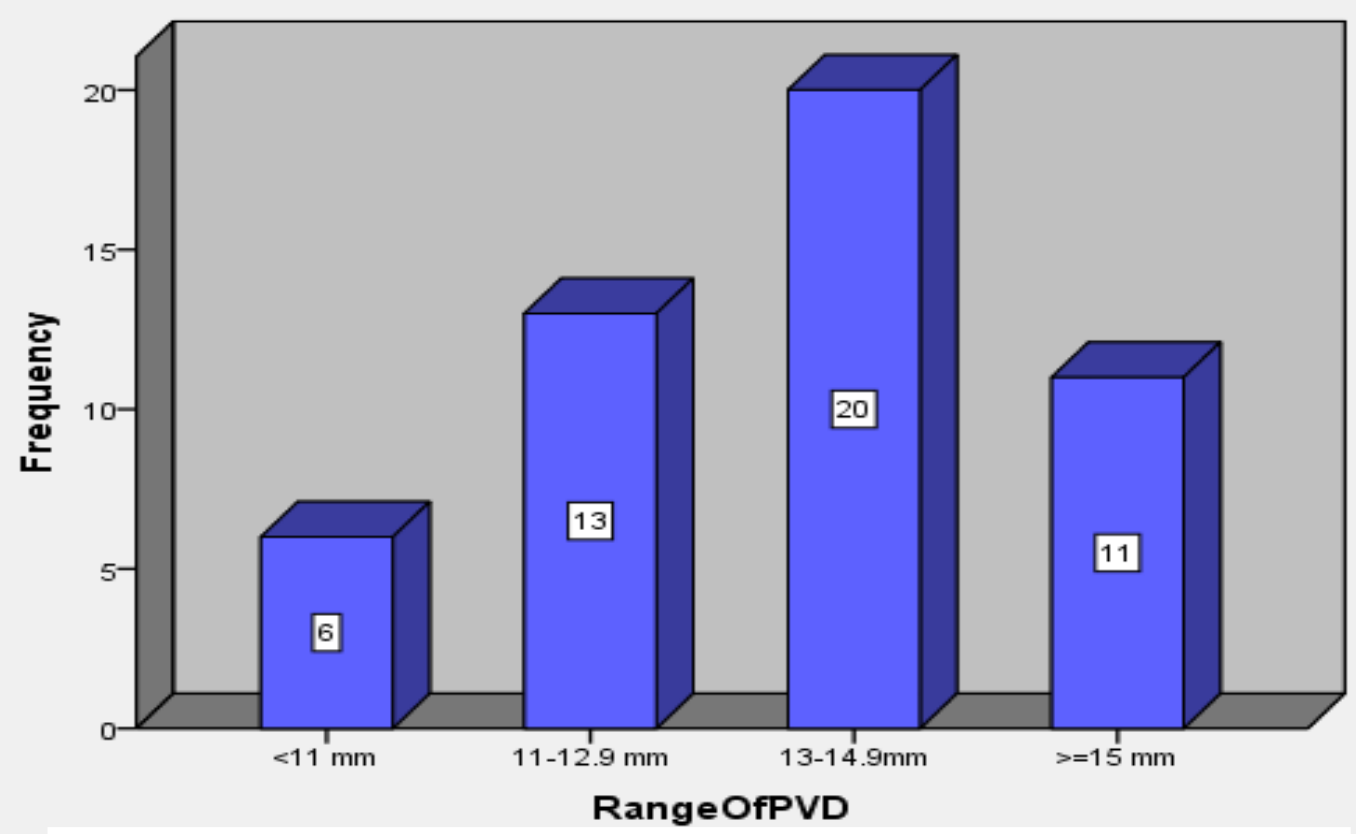

Figure 2: Portal vein diameter of the patients with varices 
Table 1: Statistical relation between pvd and varices

\begin{tabular}{|c|c|c|c|c|c|}
\hline Small or large varices & \multirow{2}{*}{$\mathrm{N}$} & \multicolumn{2}{|c|}{ Portal vein diameter (in mm) } & Std. & p-value \\
\cline { 3 - 5 } & & Mean & Median & Deviation & \\
\cline { 3 - 5 } & 3 & 10.4000 & 10.0000 & .87178 & .59936 \\
no varices & 13 & 13.2385 & 13.2000 & 0.024 \\
small varices & 34 & 16.8324 & 16.6000 & 1.26365 & \\
large varices & 50 & 15.5120 & 16.0000 & 2.31848 & \\
\hline
\end{tabular}

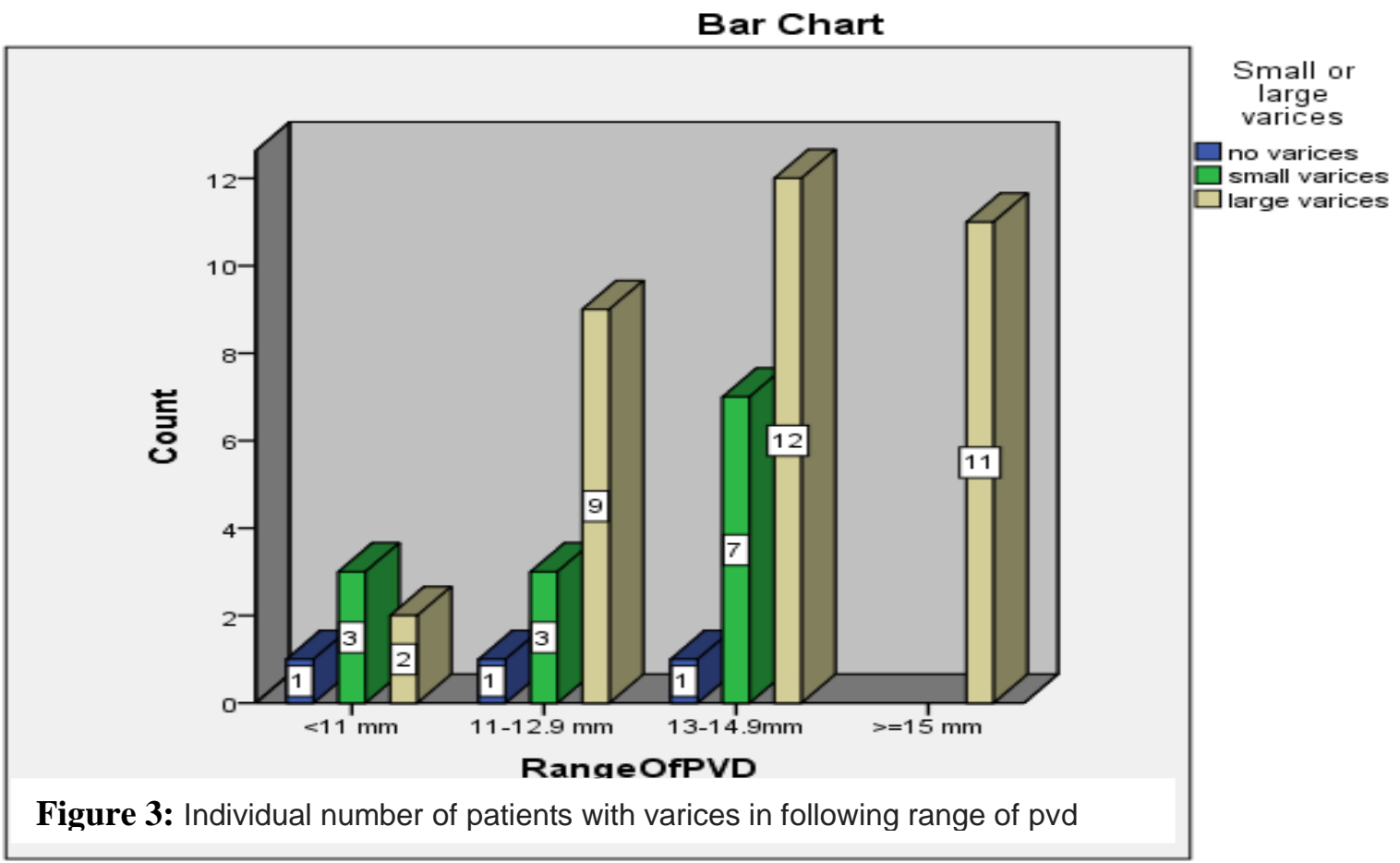

\section{DISCUSSION}

Alcohol consumption is considered as a part of culture by many races in Nepal. Due to easy availability of the alcohol, its low cost and tendency to prepare locally at home level, the excess and uncontrolled use in many unemployed population is at large. So, liver disease is of big public health importance in our country and depends largely in different cultural groups and at different periods of time. Similarly, variations can also be seen among different age groups and gender, posing significant morbidity and mortality in all classes of population. In developing countries like Nepal where people lack adequate knowledge and have poor access to health care facilities, such disease remain undiagnosed and people usually present to the hospitals in a decompensated state which has much less therapeutic benefit and carries a much grave prognosis.
Cherian et al., in their study stated that the value of the portal vein diameter over $13 \mathrm{~mm}$ could be a sensitive parameter for the prediction of presence of esophageal varices $[8,9]$. Devrajani et al., found that the mean portal vein diameter of the patients with esophageal varices is $1.4 \mathrm{~cm}$ [6]. Prihatini et al., found portal vein size of $1.5 \mathrm{~cm}$ to be predictive for variceal detection in cirrhotic patients [3]. Plestina et al., observed that portal vein size had significant diagnostic value and patients with mean portal vein size of $1.5 \mathrm{~cm}$ were at high risk of bleeding [10]. Baik et al., [11] and Franchis et al., $[12,13]$ have proposed ultrasonography for primary prevention as well as for prediction of recurrent variceal bleeding. Schepis et al., in their logistic regression study found that the portal vein diameter $>13 \mathrm{~mm}$ was an independent risk factors for the presence of varices [14]. The mean portal vein diameter in the group with esophageal varices was $12.6 \pm 2.7 \mathrm{~mm}$, while that in the group without 
esophageal varices was $10.6 \pm 2.2 \mathrm{~mm}$, with a difference of mean value of $2 \mathrm{~mm}$ [3]. Fook Hong et al, in their study, found a portal vein diameter of $1.15 \pm 0.24 \mathrm{~cm}$ among patients with esophageal varices, and $1.05 \pm 0.26 \mathrm{~cm}$ among patients without esophageal varices [15]. In Europe and the UnitedStates, they use a cutoff point of $1.3 \mathrm{~cm}$ for portal vein diameter, $12 \mathrm{~cm}$ for anteroposterior splenic measurement, and a variation for platelet count, from 80,000 to less than 150,000[3]. Ultrasonography showed that average portal vein diameter of the patients with gastro-esophageal varices was $13.998 \pm 1.123 \mathrm{~mm}$ and without gastroesophageal varices was $11.545 \pm 1.514 \mathrm{~mm}$ [3]. Portal vein diameter for development of gastroesophageal varices was $11 \mathrm{~mm}$ according to Sarwar et al., [16] study and $13.5 \mathrm{~cm}$ according to Thomopoulos et al. [17]. Mandal et al, [18] in their study found that in patients with gastroesophageal varices, grading of varices directly correlated with portal vein diameter and spleen size. $(r=0.707$ and 0.467 respectively). That implied, when portal vein diameter and spleen size increased, gastroesophageal varices also transformed to higher grades. Average portal vein diameter and spleen size in higher grade varices were $14.43 \pm 0.86 \mathrm{~mm}$ and $15.36 \pm 2.14 \mathrm{~cm}$ [18]. Thus, various studies have shown statistical significance for varying portal vein diameters correlating relationship with esophageal varices ranging from portal vein diameter $11 \mathrm{~mm}$ to $15 \mathrm{~mm}$. Our study showed that the mean diameter of the portal vein was $10.4 \mathrm{~mm}$ in the patients without varices. For the patients with small varices, the mean portal vein diameter was $13.23 \mathrm{~mm}$ and for those with large varices, the mean portal vein diameter was $16.83 \mathrm{~mm}$. The results show that the presence of varices was associated with the mean portal vein diameter of $13.23 \mathrm{~mm}$ or more. When portal vein diameter is increased, gastro-esophageal varices also transformed to higher grades. The result was statistically significant with the $\mathrm{p}$ value of 0.024 .

\section{REFERENCES}

1. Runyon BA. Management of adult patients with ascites due to cirrhosis: An update. Hepatology. 2009;49(6):2087-107.

2. Giannini E, Botta F, Borro P, Risso D, Romagnoli P, Fasoli A, et al. Platelet count/spleen diameter ratio: proposal and validation of non-invasive parameter to predict the presence of esophageal varices in patients with liver cirrhosis .Gut.2003;52:1200-5.

3. Prihatini, J, Lesmana LA, Manan C, Gani RA. Detection of esophageal varices in liver cirrhosis using non-invasive parameters. Acta Med Indones. 2005; 37(3): 126-131.
4. Bosch J, Navasa M, Garcia-Pagan JC. Portal hypertension. MedClin North Am.1989; 73: 931-53.

5. Binţinţan A. Non-invasive ultrasoundbased diagnosis and staging of esophageal varices in liver cirrhosis A systematic review of the literature published in the third Millenium.Med Ultrason. 2013;15(2): 116-124.

6. Devrajani BR, Soomro AA, Attra KM. Variceal Bleeding and its Dependence on Portal Vein Size in Liver Cirrhotic Patients. Adv. in Med. Dent.Sci.2010; 3(3): 60-60.

7. de Franchis R, Pascal JP, Burroughs AK, Henderson JM, Fleig W, Groszmann RJ, et al. Definitions, methodology and therapeutic strategies in portal hypertension: A consensus development workshop. J Hepatol.1992;15:256-261.

8. Grace ND, Groszmann RJ, Garcia-Tsao G, et al. Portal hypertension and variceal bleeding: an AASLD single topic symposium. Hepatology.1998; 28: 868880.

9. Cherian JV, Deepak N, Ponnusamy RP, Somasundaram A,Jayanthi V. Noninvasive predictors of esophageal varices.Saudi J Gastroenetrol. 2011; 17: 64-68.

10. Plestina S, Pulanie R, Kralik M, Samarzija M. Color Doppler ultrasonography is reliable in assessing the risk of esophageal variceal bleeding in patients with liver cirrhosis.Wien Klin Wochenschr.2005;20: 711-7.

11. Baik SK., Kim JW, Kim HS, Kwon SO, Kim YJ, Park JW, et al. Recent Variceal Bleeding: Doppler US Hepatic Vein Waveform in Assessment of Severity of Portal Hypertension and Vasoactive Drug Response. Radiology.2006; 240: 574-580.

12. Franchis RD. Non-invasive (and minimally invasive) diagnosis of esophageal varices. Journal of Hepatology.2008; 49(4): 52027.

13. Franchis RD. Noninvasive Diagnosis of Esophageal Varices: Is It Feasible?. The American Journal of Gastroenterology.2006;101: 2520-22.

14. Schepis F, Camma C, Niceforo D. Which patients with cirrhosis should undergo endoscopic screening for esophageal varices detection. Hepatol.2001;33: 333338.

15. Ng FH, Wang SY, Loo CK, Lam KM, Lai CW, Cheng CS. Prediction of esophagogastric varices in patients with 
liver cirrhosis. J Gastroenterol Hepatol. 1999; 14:785-90.

16. Sarwar S, Khan AA, Alam A. Nonendoscopic prediction of presence of esophageal varices in cirrhosis.J Coll Physicians Surg Pak.2005; 15 (9): 528-31.

17. Thomopoulos KC, Labropoulo KC, Mimidis KP. Noninvasive predictors of the presence of large esophageal varices in patients with cirrhosis.Dig Liver Dis.2003; 35 (7): 473-8.
18. Mandal L, Mandal SK, Bandyopdhyay D,Datta S. Correlation of portal vein diameter and splenic size with gastroesophageal varices in cirrhosis of liver.JIACM.2011; 12(4): 266-70

\section{Article History:}

Received: 2 January, 2017

Accepted: 17 January, 2017

Published online: 25 February, 2017

\section{For Citation:}

Basnet A et al. Portal Vein Diameter by Ultrasonography as a non-Invasive Predictor of Esophageal Varices, International Journal of Medicine \& Biomedical Sciences. 2017; 2(1):6-10 